\title{
Dinka Language
}

National Cancer Institute

\section{Source}

National Cancer Institute. Dinka Language. NCI Thesaurus. Code C153900.

An Eastern Sudanic Nilotic dialect cluster spoken by the Dinka people of South Sudan. 